\title{
XXIII.
}

\section{Beitrag zur Physiologie und Pathologie des Blutes $\left.{ }^{1}\right)$.}

\author{
Von Prof. F. Wilh. Zahn in Genf.
}

Functionelle Störungen der Organe in Form krankhafter Erscheinungen können eine mechanische Ursache haben, oder durch eine anormale Ernährung oder aber auch abnorme Innervation bedingt sein, d. h. es können die Ursachen derselben physikalische, chemische oder physiologische sein. Häufig wird die eine und andere Art von Ursachen gleichzeitig einwirken, zumeist indem die eine die andere zur Folge hat.

Die am häufigsten vorkommenden acuten und chronischen, localen und besonders allgemeinen functionellen Störungen und wohl auch anatomischen Veränderungen werden ohne Zweifel durch eine anormale Ernährung der Gewebe und besonders der sogenannten parenchymatösen Organe bedingt. Dieses kann entweder in der Weise geschehen, dass die Ernährungsflüssigkeit, d. h. das Blut, bestimmte, für die normale Functionirung der Gewebszellen nothwendige Substanzen nicht oder in ungenügender Menge enthält, oder dass in demselben für sie schädliche Substanzen enthalten sind. Ist letzteres der Fall, so können diese von aussen stammen oder aber im Organismus selbst gebildet worden sein und resorbirt durch das Blut den Organen zugeführt, in deren Zellen physiologische Störungen und selbst anatomische Veränderungen verursachen.

Die von aussen stammenden schädlichen, sogen. toxischen Substanzen können, weil ihr chemischer Charakter in der Regel genau gekannt ist, sei es im Inhalt der Einführungswege, sei es im Blut oder aber in den Excreten häufig verhältnissmässig leicht und sicher nachgewiesen werden.

Dies ist auch der Fall für gewisse, unter physiologischen Bedingungen im Organismus gebildete Substanzen, Excrete, wie

1) Mitgetheilt auf der deutschen Naturforscherversammlung zu Freiburgi.Br. 1883. 
die Harn- ind Gallenbestandtheile und die Kohlensäure, die anstatt aul natürlichem Woge eliminirt zu werden, in's Blut aufgenommen oder in ihm angehäuft werden. Aehnlich verhält es sich noch mit einigen unter pathologischen Verhältnissen hier entstandenen Stoffen, wie mit dem Zucker, Ammoniak und Aceton.

Ausser den letztgenannten werden im Organismus unter pathologischen Bedingungen, so namentlich in den Infectionskrankheiten, den entzündlichen und nekrotischen Prozessen und wohl auch in manchen Geschwülsten wahrscheinlich Substanzen gebildet, die auf ihre Nachbarschaft wie auch auf den Gesammtorganismus schädlich wirken können. Dieses muss wegen der hier und dort auftretenden anatomischen Veränderungen und namentlich der klinischen Erscheinungen halber theoretisch angenommen werden, da sich sonst letztere und erstere nicht gut anders erklären liessen. Thatsächlich nachgewiesen sind solche Substanzen bei den genannten pathologischen Prozessen bis jetzt allerdings noch nicht, obwohl es sicherlich nicht an Bemühungen gefehlt hat, ihr Dasein sei es anf directem oder indirectem Wege Cestzustellen.

Seitdem es gelungen ist in den Organen von an Infectionskrankheiten gestorbenen Individuen und namentlich an den primär erkrankten Stellen Mikroorganismen nachzuweisen, welche für einige dieser Krankheiten sogar bestimmt als Ursache derselben anerkannt werden müssen, schien diese Frage ihrer Lösung um einen grossen Schritt näher gerückt zu sein. In Wirklichkeit ist dies aber durchaus nicht der Fall, denn wenn auch nicht gut angenommen werden kann, dass besagte Organismen auf mechanischem Wege auf den Organismus einwirken oder indem sie lemselben durch ihre Vermehrung Nahrung entziehen, und wenn es deshalb fast nothwendigerweise angenommen werden muss, dass die oft so grossartigen Störungen bei den durch sie verursachten Krankheiten durch chemische Körper bedingt werden, so ist deren Vorhandensein aber noch nicht bewiesen, sondern nur im Hinblick auf die fermentative Wirkung ähnlicher, organische Substanzen zersetzender Organismen wahrscheinlich geworden. Ebenso wenig wie für die Infectionskrankheiten konnten in den anderen pathologischen Prozessen, für welche ebenfalls schon längst solche schädliche Stoffe aus theoretischen Erwägun- 
gen angenommen sind, dieselben in Wirklichkeit nachgewiesen werden. Die einzigen hierfür verwerthbaren Thatsachen sind die von Salkowski und Brieger gefundenen, wonach die schon unter normalen Verhältnissen im Harn in geringer Menge vorkommenden Substanzen der aromatischen Gruppe: Indol, Phenol und Kresol, die, wie Baumann und Brieger annehmen, ,auf eine fermentative Zersetzung von Eiweiss, bezw. Tyrosin zurückzuführen" seien und durch Weyl, Baumann und Nencki als Fäulnissproducte erkannt wurden, in gewissen Krankheiten vermehrt sind. E. Salkowski fand im Harn eine Vermehrung von Indol und Phenol bei Ileus, Peritonitis und in einem Fall von abdominalen Lymphosarcomen ${ }^{1}$ ). Ausserdem hat derselbe experimentell nachgewiesen, dass nach Darmunterbindung die gleichen Substanzen im Harn auftreten oder wenn sie schon vorher darin vorhanden waren, vermehrt werden und dass diese Erscheinung mit der Lösung der Ligatur schwindet; ferner hat er noch gezeigt, dass unter sonst gleichen Bedingungen diese Substanzen im Harn nicht auftraten, wenn den Thieren vorher eine Gallenfistel angelegt worden war. Salkowski, der schon auf Grund früherer Versuche ebenso wie Baumann und Nencki erkannt hatte, dass Phenol ein Product der Pancreas- resp. Darmverdauung ist, ist auf Grund dieser Untersuchung geneigt anzunehmen, dass dasselbe auch noch anderswo wahrscheinlich aus Körpereiweiss entstehen könne. Dem entgegen sind die Angaben von Hoppe-Seyler und Nencki, wonach im Blut oder in anderen thierischen Säften Phenol nicht vorkäme, dagegen aber könnte nach Nencki in Finsicht auf die Brieger'schen Mittheilungen sein Vorkommen in pathologischen Säften, wenn auch als ein seltenes, doch als erwiesen erachtet werden ${ }^{2}$ ).

Die Befunde L. Brieger's sind denjenigen Salkowski's ganz analog. Er fand eine beträchtliche Vermehrung des Phenols im Harn, namentlich bei Peritonitis, acuten Infectionskrankheiten, einem Fall von Tetanus traumat. mit Jauchung und bei Carcinose; ausserdem hat er dasselbe noch in phlegmonösem Eiter nachweisen können ${ }^{3}$ ).

1) Dieses Archiv Bd. 73. S. 409-443.

2) Centralbl. f. d. med. Wiss. 1878. No. 34.

3) Ebenda. 1878. No. 30 und Zeitschr. f. physiol. Chem. 1878. S. 241-258. 
Ueberzengt, dass die in Folge gewisser Localerkrankungen auftretenden allgemeinen Störungen durch an den primären Krankheitsheerden gebildete schädliche Substanzen chemischer Natur bedingt sein müssen, hatte ich schon vor 10 Jahren versucht einen längst bekannten, den Stenson'schen, Versuch za verwerthen, welcher sehr geeignet schien die Richtigkeit einer solchen Annahme zu beweisen. Zu jener Zeit wurden nehmlich in Laboratorium der medicinischen Klinik zu Strassburg von A. Weill) Untersuchungen über die bei diesem Versuch auftretenden Erscheinungen angestellt, wobei jedoch die Frage nach der Ursache des Allgemeintodes ganz ausser Betracht gelassen wurde. Diese gerade interessirte mich nun und da ich nirgends in der Literatur etwas darüber verzeichnet fand, stellte ich selbst Versuche an um dieselbe womöglich zu erforschen.

Comprimirt oder unterbindet man nach einer der bekannten Methoden bei Kaninchen die Aorta allein oder mit ihr auch die Vena cava unterhalb der Nierenarterien und lässt dann die Compression fortbestehen, so gehen die Thiere im Laufe des Tages zu Grunde. Noch rascher geschieht dies, wenn man nach 4-5 Stunden die Compression aufhebt resp. die Unterbindung löst und durch wiederholtes sanftes Reiben Aorta und Vena cava für den Blutstrom wieder durchgängig zu machen sucht. Gelingt dies, was aus dem Auftreten fibrillärer Zuckungen der vorher vollkommen gelähmt gewesenen Muskeln der hinteren Extremitäten zu erkennen ist, so verschlimmern sich die vorher schon vorhandenen Allgemeinerscheinungen rasch und bedeutend.

Bald nach Herstellung der Compression treten nehmlich, wie bekannt, bei einem solchen Thiere Local- und Allgemeinerscheinungen auf, die es als krank erscheinen lassen. Sein gelähmter Mintertheil liegt ausgestreckt auf einer Seite, der nicht gelähmte Vordertheil sitzt regungslos aufrecht, seltene und nur langsame Bewegungen ausführend; vorgelegtes Futter wird nicht angerihhr, das Athmen ist beschleunigt, die Herzaction ist geschwächt und die Temperatur, in Mund und Mastdarm gemessen, wird mehr and mehr subnormal.

Nach Wiederherstellung der Circulation steigern sich, wie

7) Ber Stenson 'sche Versuch. Inaug.-Diss. Strassburg 1873. 
gesagt, diese Symptome beträchtlich. Bald danach hält sich das Thier kaum mehr auf den Vorderbeinen, der Kopf sinkt etwas nach vorn über, die Augen sind halb geschlossen, die Conjunctiven werden blasser, die Pupillen erweitern sich, reagiren erst nur noch schwach und später gar nicht mehr auf Lichteinfall, die Respiration wird oberflächlicher, die Herzcontractionen sind unfühlbar, die Temperatur sinkt immer mehr und geht selbst bis auf $27^{\circ} \mathrm{C}$. herunter, das Thier reagirt immer weniger und schliesslich gar nicht mehr auf Hautreize, nur die Conjunctiven bleiben. noch etwas empfindlich, es lässt den Kopf seitwärts sinken, bald folgt diesem auch der Rumpf und nach 3-4 Stunden ist es todt. Macht man nun sofort die Section des Thieres, so findet man nur die Muskeln der hinteren Extremitäten blasser als normal, das Herz in Diastole und besonders rechts gefüllt mit viel flüssigem, nicht zu Gerinnung neigendem dunklem Blute. An den übrigen Organen finden sich keine besonderen Veränderungen. Ich hatte früher lange nach mikroskopischen Veränderungen der parenchymatösen Organe, besonders des Herzens geforscht, ohne jedoch die gewünschten Resultate zu erzielen; es gelang mir dies auch nicht, wenn ich die Thiere möglichst lang am Leben zu erhalten suchte, da dies doch nie länger als 30 Stunden nach Beginn der Operation sein konnte.

An Mittel und Wegen, die vermuthlich im gelähmten und abgestorbenen Hintertheil der Thiere entstandenen und durch die Lymph- oder, bei wiederhergestelltem Kreislauf, Blutgefässe dem Gesammtorganismus zugeführten schädlichen Substanzen nachzuweisen, fehlte es mir damals gänzlich und erst als ich im Frühjahr 1883 gelegentlich anderer Untersuchungen meinem Freunde und Collegen A. Danilewsky meine Vermuthungen bezüglich der Existenz solcher Substanzen im kranken Thierkörper mittheilte und ihn befragte, ob es nicht Methoden gäbe, dieselben eventuell im Blute nachzuweisen, hatte derselbe die Güte mir eine Methode anzugeben, mittelst welcher es ihm bereits gelungen war, in anderen thierischen Substanzen das Vorhandensein von, der aromatischen Gruppe angehörigen Körpern nachzuweisen. Die damals noch unbekannte Methode war keine andere als die jetzt sogenannte und von mehreren Forschern zu ähnlichem Zweck angewandte Azoreaction. Herr Danilewsky 
rieth mir um so mehr dazu, dieselbe bed meinen Cntersuchungen zu verwerthen, als nach seiner Erfahrung im normalen arteriellen Blut keine Substanzen vorhanden sind, die mit dem Azoreactiv die betrefiende, sehr charakteristische Reaction geben. Der mir von ihm vorgeschlagene Gang der Untersuchung ist der folgende: das ganz frische, soeben den Gefüssen entnommene Blut, mindestens $5 \mathrm{ccm}$, wird mit wenig Essigsäure neutralisirt, dann sofort mit einer der Blutrgenge gleich grossen Menge Alkohol versetzt, die Mischung auf dem Wasserbad zur Trockne eingedampft, der Rückstand möglichst fein zerkleinert, mit Alkohol übergossen, bis zum Kochen erhitzt und filtrirt; der in der Schale zurïckbleibende Rückstand wird nochmals mit gleichviel Alkohol übergossen, wieder erhitzt und filtrirt. Beide Filtrate zusammen werden evaporirt, mit Aetheralkohol ausgezogen, filtrirt und da das Filtrat sauer reagirt mit wenig Kali- oder Natronlauge neutralisirt. Zum Nentralisiren darf, wie ich mich durch mehrere Misserfolge überzeugte, kein Ammoniak verwandt werden, da sonst später wegen der Anwesenheit von Ammoniaksalzen die Azoreaction auch in solchen Flüssigkeiten ausbleibt, die wie der Harn sie stets und sehr deutlich geben. Das nunmehr neutrale Aetheralkoholfiltrat wird zur Trockne gebracht and der schmierige, bräunliche Rückstand mit heissem Wasser ausgezogen. Dieses Extract wird dann filtrirt und das klare Filtrat auf Zucker und Pepton geprüt. Bei Abwesenheit beider wird dem Filtrat etwas Weniges von dem Azoreactiv zugesetzt, die Mischung mit Salzsäure schwach angesäuert und nach $\frac{1}{4}-\frac{1}{2}$ Minute bis zu schwach alkalischer Reaction mit Natronlauge versetzt. Sind in dem solchermaassen behandelten Extract Substanzen vorhanden, die den Oxy- oder Amidoderivaten des Benzolkerns angehören, so nimmt je nach ihrer Menge die Flïssigkeit sofort oder doch nach wenigen Minuten eine orange-rothe, gewöhnlich sogar orangedunkelrothe Färbung an ${ }^{1}$ ).

Zur eigenen Uebung und zur Controle für die späteren Versuche untersuchte ich auf diese Weise zuerst Blut aus der Carotis, Vena jugularis und mesenterica und aus dem rechten

:) Die detaillirte Beschreibung der Reaction und ihrer Anwendungen findet sich in einer Mitheilung von A. Danilewsky in den Arch. des scienc. physiques et natur. Jars 1884. 
Herzen grosser, kräftiger, gesunder Kaninchen. Hierbei ergab sich die nicht erwartete Thatsache, dass bei dem gleichen Thier das Extract von Carotisblut gar keine, venöses Blut aus der V.jug., selten schwache, solches aus dem rechten Herzen etwas häufiger schwache und endlich Mesenterialvenenblut immer sehr deutliche Azoreaction gab. Aus diesem Befund geht hervor, dass im Mesenterialvenenblut regelmässig Substanzen vorkommen, welche die gleiche Reaction geben wie die oben erwähnten, den Oxy- oder Amidoderivaten des Benzolkerns angehörigen Substanzen. Ferner zeigt er auch, dass diese Substanzen ganz oder doch zum grössten Theil in der Leber zerstört oder aber durch sie ausgeschieden werden und dass die in geringen Mengen im Blut des rechten Herzens vorkommenden ähnlichen oder gleichen Stoffe wohl in der Lunge ihren Untergang finden. Bereits vor mir hat A. Danilewsky schon zuweilen Spuren derselben im Extract des Körpervenenblutes gefunden, eine Thatsache die durch obigen Befund nur bestätigt wird.

Nachdem diese Thatsachen durch mehrmals wiederholte Versuche festgestellt waren, ging ich daran, in gleicher Weise das Blut von Thieren zu untersuchen; welchen vorher die Aorta allein oder die Aorta und Vena cava infer. unterbunden worden war. Die Unterbindung dieser Gefässe nahm ich zuletzt immer nur von der Bauchhöhle aus vor. $Z$ u diesem $Z_{w}$ eck wurde dem Thier die Bauchhöhle in der Mittellinie eröffnet und nachdem die Därme beiseite gelegt waren, wurden unterhalb der Nierenarterien nach innen von den Ureteren zwei lange, starke, gerade Nadeln, in deren Oesen sich die beiden Enden eines dicken Seidenfadens eingefädelt fanden, durch die Rückenmusculatur und Haut gestossen und dann aussen gefasst und angezogen bis innen die Schlinge gerade um die Gefässe und Wirbelsäule lag. Hierauf wurden die Därme in die richtige Lage zurückgebracht und die Bauchwunde geschlossen. Nachdem ich mich nun überzeugt hatte, dass die Thiere sich ganz normal verhielten, wurden die beiden Fadenenden fest angezogen und über einem Kork festgebunden. Sobald dies geschehen war, traten auch rasch die bekannten localen Erscheinungen des Stenson'schen Versuches auf, nehmlich Verlust der Sensibilität und Motilität der hinteren Extremitäten, Stuhlentleerung, Harnverlust und bei Böcken 
Erection. Bei manchen Thieren verlor sich allmählich die anfänglich vorhandene vollständige Lähmung und zwar wahrscheinlich durch Herstellung eines von Stannius schon nachgewiesenen Collateralkreislaufs durch die Aa. epigastr. sup. und inferior, bei anderen blieb sie vollständig bestehen. Von diesen Thieren wurden die einen nach 5-6 Stunden durch Blutentziehung aus der Carotis und Vena jugularis, manche auch durch Schlag in's Genick zur Gewinnung des Herzblutes getödtet; bei anderen wurde nach 3 bis 4 Stunden die Ligatur gelöst, indem der Faden aufgeknotet und abwechselnd vorsichtig und langsam nach der einen und anderen Seite gezogen wurde. Sodann wurde der Faden auf einer Seite dicht an der Haut durchschnitten und auf der anderen Seite ganz herausgezogen. Schliesslich wurde die ligaturstelle von der Bauchseite her sanft gerieben und das Thier nach einiger Zeit sich selbst überlassen. Mitunter erfolgte hierauf gar nichts Besonderes und die spätere Section zeigte, dass an der Ligaturstelle Verklebung der Gefässwand und Thrombenbildung bestanden, welche die Wiederherstellung der Circulation verhindert hatte, bei anderen jedoch, und zwar der grösseren Anzahl, hatte sich diese wieder eingestellt und bei diesen wurden dann die oben geschilderten schweren und rascher verlaufenden Allgemeinerkrankungen beobachtet.

Die Untersuchung des Blutextractes aller dieser Thiere ergab folgende Resultate: das arterielle und venöse Blut von Thieren, welche nur vorübergehend gelähmt waren, zeigte nur schwache Azoreaction. Deutlicher war sie schon bei solchen Thieren, bei denen die Lähmung vollständig war und welche ohne vorherige Lösung der Ligatur getödtet worden waren oder welche man nach der Lösung derselben hatte langsam absterben lassen. Noch deutlicher aber war sie bei denjenigen Thieren, die bald nach Lösung der ligatur und Wiederherstellung des Kreislaufes in den hinteren Extremitäten getödtet worden waren. Bei allen Thieren war die Reaction stets am stärksten im Extract des Blutes des rechten Herzens.

Hieraus geht hervor, dass in Folge von partiellem und besonders vollständigem Absterben von Geweben bereits während des Lebens Substanzen im Blut auftreten können, die mit Ausnahme des Mesenterialvenenblutes sich für gewöhnlich nicht oder doch nur in Spuren darin finden. 
Mehrere Male ergaben diese Blutuntersuchungen vollständig negative Resultate. Für die Méhrzahl derselben konnte ich den Grund des Misslingens auffinden und zwar bestand er darin, dass ich zum Neutralisiren des sauern Extractes Ammoniak verwandt hatte und, wie ich mich durch Controlversuche mit Harn überzeugte, dessen Anwesenheit das Eintreten der Reaction verhindert. In 3 Fällen hatte ich aber auch ohne nachweisbaren Grund im Extract des Mesenterialvenenblutes die Reaction nicht erhalten und ebenso $2 \mathrm{Mal}$ nicht beim Stenson'schen Versuch im Extract des Gesammtblutes.

Um sicher zu sein, dass beim Stenson'schen Versuch nicht das Trauma und der dadurch bedingte entzündliche Prozess die erwähnte Blutveränderung bedinge, habe ich bei $3 \mathrm{Ka}$ ninchen den Bauchschnitt und die Umschnürung der Wirbelsäule, aber mit Auslassung der Gefässe, ganz ebenso wie bei jenem vorgenommen. Die so behandelten Thiere verhielten sich vollkommen normal und die spätere Blatuntersuchung ergab insofern ein negatives Resultat, als es sich nicht anders verhielt als dasjenige vollkommen normaler Thiere.

Die geringe Blutmenge, welche vom Kaninchen erhältlich ist, $20-30 \mathrm{~cm}$, und meine geringe Uebung in chemischen Untersuchungen, haben mir nicht erlaubt die wichtige Frage zu lösen, welcher Natur der-oder diejenigen Stoffe sind, welche die Azoreaction im Extract des normalen Mesenterialvenenblutes oder im Gesammtblut nach vorheriger Aortenunterbindung geben. Wäre es erlaubt, auf Grund der erwähnten Farbenreaction eine Vermuthung darüber auszusprechen, so möchte ich annehmen, dass der- oder diejenigen Substanzen, welche sie geben, zu den Oxyoder Amidoderivaten des Benzolkerns gehören.

Ebenso wenig gelang es mir festzustellen, ob der oder die qualitativ im Gesammtblutextract nachweisbaren Substanzen auch gerade diejenigen sind, welche beim Stenson'schen Versuch, sei es ohne oder nach vorheriger Lösung der Gefässligatur, die so schweren Allgemeinerscheinungen hervorrufen. Ich habe gesunden Kaninchen solches Extract injicirt ohne dass dieselben danach die geringsten Erscheinungen zeigten, dagegen wurden Frösche, welchen dasselbe in den Rückenlymphsack injicirt wurde in ihren Bewegungen langsamer und sassen meistens eine ge- 
raume Zeit regungslos da, jedoch dauerte dieser Zustand nicht lange und sie erholten sich wieder vollständig. Diese Versuche beweisen also wenig oder auch gar nichts.

Um die mir gestellte Frage definitiv zu entscheiden, hatte ich mich auch eine Zeit lang bemüht festzustellen, ob nicht etwa nach der Aortenunterbindung in Folge des Absterbens von der Ciroulation entzogenen Geweben ptomainähnliche Körper gebildet würden, die in's Blut aufgenommen auf den Organismus toxisch wirken könnten. Jedoch anch in dieser Hinsicht hatte ich nur negative Resultate zu verzeichnen, möglicherweise, wie ich glaubte, wegen der geringen Menge des Materials womit ich operirte. Ich stand deshalb auch bald davon ab solche nachweisen zu wollen und besonders noch weil nach den Untersuchungen ron Guareschi und $\mathrm{Moss0}$ ) die meisten bisherigen Angaben hierüber einen nur sehr zweifelhaften Werth haben und weil, nach den Mittheilungen Cappola's ${ }^{2}$ ) die sogenannten Ptomaine nicht nur nicht im lebenden Organismus, sondern auch nicht einmal bei der Fäulniss entstehen, sondern ein Product der chemischen Manipulationen (Stas-Otto'sches Verfahren) sind.

Somit habe ich durch die vorstehenden Untersuchungen meinen Eingangs erwähnten Zweck nicht vollständig erreicht und es ist mir nicht gelungen die vermutheten toxisch wirkenden Sabstanzen im Blut aufzufinden. Immerhim haben dieselben doch gezeigt, dass bei einfacher Gewebsnekrose in Folge von Circulationsstörung im abgesperrten Blute, was mir jedoch aus ein anderes $\mathrm{Mal}$ zu erwähnenden Gründen nicht wahrscheinlich ist, oder in den übrigen der Circulation entzogenen Geweben chemische Körper entstehen und dem Gesammtblut in solcher Menge zugeführt werden können, dass sie in dem Extract desselben deutlich nachweisbar sind.

Ferner ergab sich aus den Blutuntersuchungen normaler Thiere ausser der Verschiedenheit der Azoreaction für das Extract der verschiedenen Blutarten die andere wichtige Thatsache, dass in der Leber die dieselben gebenden Substanzen ganz oder loch grossentheils zerstört werden können. Dieses würde somit

1) Archives ital. de Biologie. Tom. II, p. 373.

3) Gazetta chim. Italiana. T. XIT. 1882. 11. ibid. 'T. XIII. 1883. S. a. Areh. ital. de Biol. T.IV. p. 63. 
eine frühere Beobachtung von Schiff bestätigen, wonach gewisse toxische Substanzen, wie z. B. Nicotin und Hyoscyamin in diesem Organ unschädlich gemacht werden können ${ }^{1}$ ).

\title{
XXIV.
}

\section{Untersuchungen über das Vorkommen von Fänlnisskeimen im Blut gesunder Thiere.}

\author{
Von Prof. F. Wilh. Zahn in Genf.
}

Es könnte fast überflüssig erscheinen neue Versuche über das Vorkommen von Fäulnisskeimen im gesunden Thierkörper anzustellen und zu veröffentlichen, dermaassen häufig ist bereits diese Frage ventilirt worden. Aber obgleich dieselbe von vielen ausgezeichneten Forschern discutirt wurde, kann sie dennoch keineswegs als abgeschlossen betrachtet werden. Während nehmlich die Einen auf Grund ihrer Untersuchungen zu dem Schluss kamen, dass in den Geweben und Flüssigkeiten gesunder Thiere niemals solche Keime vorhanden sind, kamen die Anderen auf Grund ebenso gewissenhafter und sorgfältig angestellter Versuche zu einem geradezu entgegengesetzten Resultat; eine dritte Reihe endlich hatte mit der stets gleichen Methode bald negative, bald positive Ergebnisse, wodurch ihnen die Entscheidung, auf welche Seite sie sich schlagen sollten, jedenfalls schwer fallen musste. So erging es z. B. auch mir, als ich bald nach dem Erscheinen der Tiegel'schen Mittheilungen ${ }^{2}$ ), dessen Versuche wiederholte und wie er bald positive, bald negative Resultate hatte. Obwohl überzeugt, dass selbst ein einziger Versuch mit negativem Resultat, d. h. ohne ein Auftreten von Fäulnisskeimen unter sonst scheinbar gleichen Bedingungen, mehr beweise als viele positive, d. h. mit Auftreten solcher, konnte ich mich doch nicht entșchliessen, meine damaligen Ver-

1) M. Schiff, Arch. des sciences phys. et natur. 1877. p. 293.

2) E. Tiegel, Coccobacteria septica im gesunden Wirbeltbierkörper. Dieses Archiv Bd.60. S. 459. 1875. 